\title{
Implementação de teleatendimento em saúde mental para estudantes de Medicina durante a pandemia da Covid-19
}

\author{
Implementation of a telemental health service for medical students during the COVID-19 pandemic
}

\author{
Suzana Pacheco Liberal' ${ }^{1}$ | |spl.suzana@gmail.com \\ Geovani Bordiano' (D) giovannibordiano@gmail.com \\ Giovanni Marcos Lovisi' (D) giovannimlovisi@gmail.com \\ Lucia Abelha' (1) lucia.abelha@gmail.com

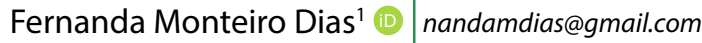 \\ Camila Oliveira Carvalho' (1) camilaoliveira@hucff.ufrj.br \\ Lina Rosa Nunes Morais' ${ }^{1}$ (D) linarnm@hotmail.com \\ Marco Antonio Alves Brasil' ${ }^{1}$ (D) marcoantonioabrasil@gmail.com
}

\section{RESUMO}

Introdução: A pandemia provocada pela Covid-19 pode ser considerada um evento estressante grave e desencadear repercussões negativas na saúde mental dos estudantes de Medicina, como sofrimento psíquico e desenvolvimento ou agravamento de transtornos mentais, trazendo prejuízos à vida acadêmica, social e profissional desses alunos. Em consequência da interrupção das aulas e do distanciamento social preconizado pelos órgãos de saúde durante a pandemia, o Setor de Atendimento em Saúde Mental destinado aos alunos de Medicina da Universidade Federal do Rio de Janeiro (UFRJ) necessitou cancelar os atendimentos presenciais do Hospital Universitário Clementino Fraga Filho no início de março de 2020 e pensar em outras formas de cuidado em saúde mental para esses alunos.

Relato de experiência: Trata-se de um relato de experiência acerca da implementação do teleatendimento em saúde mental destinado aos estudantes de Medicina da UFRJ durante a pandemia, para a continuidade do cuidado em saúde mental de forma remota, iniciado no final de março de 2020 . $O$ atendimento está sendo ofertado por uma equipe de cinco psiquiatras, uma psicóloga e uma assistente social, todos funcionários da universidade.

Discussão: $O$ teleatendimento tem servido como um espaço importante de escuta e acolhimento diante das demandas psicossociais desses alunos e tem como desafio ultrapassar algumas barreiras que dificultam o acesso e a disponibilidade de serviços de saúde mental no campus universitário, incluindo a preservação da relação médico-paciente, a garantia da confidencialidade e qualidade, e a oferta de um espaço de cuidado em saúde mental quando a presença física não é possível.

Conclusão: Apesar das dificuldades inerentes ao rápido processo de implementação desse serviço, percebe-se o potencial da tecnologia em auxiliar a população nesse momento crítico, em especial na atenção à saúde mental de grupos específicos como os estudantes de Medicina. O teleatendimento representa um potencial de aprendizado e mudança nas formas de como o acesso ao cuidado é ofertado, com a perspectiva de trazer benefícios à saúde mental dos estudantes, mesmo após o período atual da pandemia, com a meta de expansão desses atendimentos para outros cursos da UFRJ.

Palavras-chave: Estudantes de Medicina; Covid-19; Transtornos Mentais; Telemedicina.

\section{ABSTRACT}

Introduction: The COVID-19 pandemic can be considered a severely stressful event and trigger negative repercussions on the mental health of medical students, such as psychological distress and the development or worsening of mental disorders, harming the academic, social and professional life of these students. As a result of the interruption of classes and the social distancing measures advocated by health agencies during the pandemic, the mental health care sector for medical students at the Federal University of Rio de Janeiro (UFRJ) needed to cancel the face-to-face care at the Clementino Fraga Filho University Hospital in early March 2020 and think of other forms of mental health care for these students.

Experience report: This is an experience report about the implementation of telemental health care for medical students at UFRJ during the pandemic, for the continuity of mental health care program using remote assistance, started in late March 2020 . The service is being offered by a team of five psychiatrists, a psychologist and a social worker, all university employees.

Discussion: The teleservice has served as an important space for listening and embracement in face of these students' psychosocial demands, whose challenge consists in overcoming some barriers that hinder the availability of and access to mental health services on the university campus, including the preservation of the doctor-patient relationship, the guarantee of confidentiality and quality, and the offer of a space for mental health care when the physical presence is not possible.

Conclusion: Despite the difficulties inherent in the rapid process of implementing this service, the potential of technology to help the population at this critical moment is perceived, especially regarding the attention to the mental health of specific groups, such as medical students. The telehealth represents a potential for learning and change in the ways how the access to care is offered, with the perspective of bringing benefits to the students' mental health, even after the current period of the pandemic, with the goal of expanding these services to other courses of the UFRJ. Keywords: Medical Students; Coronavirus Infections; Mental Disorders; Telemedicine.

1 Universidade Federal do Rio de Janeiro, Rio de Janeiro, Rio de Janeiro, Brasil.

Editora-chefe: Rosiane Viana Zuza Diniz. Editora associada: Izabel Cristina Meister Martins Coelho.

Recebido em 13/09/20; Aceito em 29/08/21. Avaliado pelo processo de double blind review. 


\section{INTRODUÇÃO}

A pandemia da coronavirus disease 2019 (Covid-19) pode ser considerada um evento traumático em razão do potencial de desencadear sofrimento psíquico e causar reações como extremo medo de contágio, raiva, incerteza, insônia, malestar, além do surgimento ou agravamento de transtornos mentais, como ansiedade, depressão, reações agudas ao estresse e transtorno do estresse pós-traumático. Pode ainda estar associada a comportamentos de risco, como o uso de tabaco e álcool, e ao aumento do risco de suicídio ${ }^{1-4}$. Diante desse cenário, diversos grupos estão sendo considerados mais suscetíveis às consequências psicossociais da pandemia, como é o caso dos estudantes de Medicina ${ }^{5}$.

Considerando que esses estudantes compõem parte significativa da força de trabalho dos sistemas de saúde, faz-se necessário refletir sobre a qualidade e a homogeneidade de sua formação, assim como a manutenção de sua saúde mental, que pode ser comprometida diante da atual emergência sanitária 5 . A vivência de um momento de incertezas, com potenciais repercussões profissionais para esses estudantes, coloca-os em uma posição de vulnerabilidade psicológica. Soma-se a isso o fato de que vários estudos, anteriores ao período de pandemia, sugerem que os estudantes de Medicina estão em grande risco de desenvolver problemas de saúde mental. Uma metanálise envolvendo estudos de 43 países identificou 27,2\% de prevalência de depressão ou sintomas depressivos e uma taxa de ideação suicida de $11 \%$ entre os estudantes de Medicina ${ }^{6}$.

Diante de situações de pandemia e desastres em larga escala, uma das recomendações para enfrentamento, de acordo com protocolos internacionais, envolve ofertar canais alternativos de atendimento (aplicativos, websites, telefone) ${ }^{7}$. Nesse cenário, muitos países adotaram como recurso o teleatendimento em saúde mental, que apresenta uma capacidade singular de oferecer apoio, mantendo o distanciamento social. O teleatendimento consiste na utilização das tecnologias de informação e comunicação (TIC), como telefonemas, e-mails, videoconferências e sistemas de consulta eletrônica, para oferecer atendimento em saúde de forma remota ${ }^{8}$.

Entre os potenciais benefícios do teleatendimento, está a possibilidade de as tecnologias de comunicação conectarem as pessoas e fortalecerem sua rede de apoio, o que pode trazer benefícios na prevenção do sofrimento psíquico, especialmente em situações de distanciamento social, como é o caso da pandemia da Covid-199. Outros fatores positivos incluem economia de recursos, possibilidade de acesso aos especialistas em regiões distantes, colaboração de profissionais mais experientes com os menos experientes em situações de emergência e o não deslocamento dos pacientes ${ }^{8,10,11}$. São relatadas também algumas vantagens, como: limitação da transmissão viral e proteção das pessoas que são de grupos considerados como de risco; aumento da compreensão sobre a dinâmica familiar; melhoria do acesso a pacientes que apresentam condições que interfiram em sua habilidade de sair de casa; aumento da privacidade e da sensação de segurança ao reduzir a necessidade de ir a uma instituição de saúde mental; melhora na organização da agenda médica, pois aumenta a facilidade de marcação de consultas e diminui a taxa de não comparecimentos. Em contrapartida, há algumas limitações relacionadas aos aspectos tecnológicos (como aumento da chance de interrupções por falhas técnicas), ao acesso limitado de alguns grupos populacionais a esse tipo de atendimento (idosos, pessoas que não possuem acesso à tecnologia, pessoas com deficiências visuais, auditivas e/ou enxaqueca) e ainda à ausência do contato físico, o que impede a realização do exame físico necessário em algumas condições, dificulta a utilização da comunicação não verbal e pode prejudicar o estabelecimento da relação médico-paciente ${ }^{12}$.

No cenário mundial, já se observavam, antes do advento da pandemia, crescentes investimentos e pesquisas em teleconsultas, com destaque para a Inglaterra e os Estados Unidos $^{8}$.Osteleatendimentospoderiam ser realizadosemcaráter aditivo, alternativo ou parcialmente substitutivo ao tratamento presencial e, de forma geral, demandavam uma primeira consulta presencial ${ }^{8}$. No contexto da pandemia, diversos países têm utilizado as TIC para rastreio e monitoramento de pacientes, acompanhamento de doenças crônicas, aconselhamentos e terapias (como telerreabilitação para crianças e idosos) ${ }^{10}$. Com relação à saúde mental, as teleconsultas podem ser realizadas para acompanhamento de pessoas previamente atendidas no serviço, mas também para dar suporte a novos casos que surgiram no contexto da pandemia. Alguns países como a China e Austrália têm ofertado serviços de psicoeducação, aconselhamento, supervisão e treinamento por meio de plataformas on-line, tendo como público-alvo os profissionais da linha de frente, pacientes com Covid-19 e seus familiares ${ }^{13}$.

No Brasil, destaca-se o Programa Telessaúde Brasil Redes. Criado em 2011 por algumas universidades em parceria com o governo federal, esse programa promove a integração das equipes de Saúde da Família com os centros universitários de referência em teleatendimento e incentiva a qualificação da assistência na atenção primária ${ }^{14}$. Outro exemplo é o Projeto TelePSI, ofertado pelo Ministério da Saúde em parceria com o Hospital de Clínicas de Porto Alegre (HCPA). Esse projeto configura-se como um serviço de atendimento gratuito a profissionais de saúde que estão na linha de frente da Covid-19e conta com o apoio de profissionais de psicologia e psiquiatria ${ }^{15}$.

Com o surgimento da pandemia, tornaram-se urgentes a expansão de serviços de teleatendimento e a regularização 
deles. O Ministério da Saúde publicou a Portaria n 467/2020, que dispõe sobre as ações de telemedicina na operacionalização das medidas de enfrentamento da pandemia, em caráter de excepcionalidade, autorizando sua prática nos âmbitos público e privado ${ }^{16}$. Em seguida, foi aprovada a Lei no 13.989/2020, que autoriza o uso da telemedicina em quaisquer atividades da área de saúde no Brasil, incluindo a teleconsulta, enquanto durar essa emergência sanitária ${ }^{17}$. Nesse contexto, o Conselho Federal de Medicina (CFM) ${ }^{18}$ reconheceu, por meio do Ofício n 1756/2020, a possibilidade e eticidade da utilização da telemedicina, "em caráter de excepcionalidade e enquanto durar a batalha de combate ao contágio da COVID-19" (p. 1), nas modalidades de teleorientação, telemonitoramento e teleinterconsulta. $\mathrm{O}$ Conselho Federal de Psicologia (CFP) ${ }^{19}$ resolveu, por meio da Resolução n 4/2020, autorizar a realização de teleconsulta para todos os psicólogos mediante algumas orientações, mesmo aqueles que ainda não tenham sido aprovados na plataforma e-Psi por seus Conselhos Regionais. O Conselho Federal de Serviço Social (CFESS) ${ }^{20}$ divulgou nota sobre o exercício profissional diante da pandemia do coronavírus,ressaltando que ainda não regulamentou resolução para esse tipo de atendimento, porém resguarda a autonomia profissional do assistente social para definir a forma de atendimento mais adequada, destacando a excepcionalidade diante da situação de pandemia em que se encontra o país.

Diante desse cenário, o presente artigo tem como objetivo relatar a experiência da implementação de teleatendimento em saúde mental destinado aos estudantes de Medicina da Universidade Federal do Rio de Janeiro (UFRJ) no cenário da pandemia da Covid-19.

\section{RELATO DE EXPERIÊNCIA}

O aumento da demanda espontânea de alunos de Medicina para o atendimento à saúde mental no Serviço de Psiquiatria e Psicologia do Hospital Universitário Clementino Fraga Filho (HUCFF), assim como a demanda de professores de diferentes cursos da UFRJ que procuravam ajuda para lidar com a problemática apresentada por vários alunos, motivou a reitoria da UFRJ a criar um grupo de trabalho para elaborar uma política de atenção à saúde mental do estudante da UFRJ $^{21}$. Como resultado desse trabalho, foi iniciado um projeto de atendimento aos estudantes da Faculdade de Medicina da UFRJ, posto em prática em maio de 2018, com o apoio de profissionais do Serviço de Psiquiatria e Psicologia do HUCFF e do Instituto de Estudos em Saúde Coletiva (IESC/UFRJ). A partir de julho de 2019, o projeto passa a contar com o apoio também de uma profissional do Serviço Social do HUCFF. Localizado no nono andar (sala 9E28) do HUCFF, o Setor de Atendimento Psiquiátrico e Psicológico destinado aos alunos da Faculdade de
Medicina foi oficializado em 7 de março de 2019 pela Portaria do Boletim UFRJ (BUFRJ) $n^{\circ} 10^{22}$.

Em meados de março de 2020, com o início da pandemia da Covid-19, houve a interrupção das aulas na universidade e, consequentemente, dos atendimentos aos estudantes. A partir de então, os profissionais do Setor de Atendimento Psiquiátrico e Psicológico, por meio de reuniões virtuais, iniciaram a discussão sobre o prolongamento das medidas restritivas e o isolamento social, que possivelmente estariam causando impactos emocionais aos alunos. Seria relevante, portanto, organizar uma proposta de apoio on-line aos estudantes durante a pandemia.

A análise de estudos nacionais e internacionais, a realidade social e econômica de parte significativa dos estudantes, a interrupção do primeiro semestre e a perspectiva de perder também o segundo semestre, e possíveis dificuldades familiares geradas pelo isolamento social trouxeram a preocupação com o desencadeamento, a recorrência e piora de sofrimento psíquico entre os alunos, principalmente entre aqueles com histórias preexistentes de transtornos mentais. Além disso, a pandemia poderia trazer como consequência o aumento de problemas mentais especialmente em alunos com maior vulnerabilidade psíquica ${ }^{23}$.

A partir de uma reunião com o diretor da Faculdade de Medicina, foi organizado um protocolo de atendimento remoto aos alunos que necessitassem de apoio durante o período de pandemia. Inicialmente, encaminhou-se uma mensagem acerca da oferta de atendimento on-line aos alunos da Faculdade de Medicina da UFRJ por meio do Sistema Integrado de Gestão Acadêmica (SIGA). Os alunos interessados nos atendimentos entram em contato diretamente com a secretária do programa por e-mail, a qual encaminha os contatos deles para os profissionais da equipe do projeto: cinco psiquiatras, uma psicóloga e uma assistente social, com disponibilidade semanal inicial de quatro horas cada um.

O profissional entra em contato com o aluno interessado para agendar o atendimento on-line, que poderá ser feito por meio de diversos aplicativos, como WhatsApp, Zoom e Skype, e tecnologia móvel, como telefone celular e outros, de acordo com a disponibilidade de cada estudante. Cada atendimento tem duração média de uma hora. Nele, o profissional segue condutas preestabelecidas para lidar com eventos traumáticos como orientações de mecanismos de coping (relaxamento muscular, exercícios físicos, ioga, mindfulness) e apoio social ${ }^{24}$.

São previstos inicialmente quatro atendimentos. Se for preciso, o profissional estenderá o cuidado pelo tempo necessário. Em caso de emergências psiquiátricas, será acionado o plantonista do Serviço de Psiquiatria e Psicologia 
do HUCFF. Os alunos que necessitarem de um apoio mais efetivo na área social contarão com o apoio da assistente social. Esse apoio é oferecido principalmente aos alunos ingressantes por meio de ações afirmativas e aos originários de outros estados do Brasil ou de outros países, como orientações para adquirirem benefícios disponíveis na universidade através da política de assistência estudantil (Figura 1). No primeiro semestre de 2018, 45,29\% dos alunos ingressaram na UFRJ por meio de ações afirmativas, provenientes de escolas públicas, dos quais $21 \%$ faziam parte de ações afirmativas de renda (renda familiar per capita abaixo de um salário mínimo e meio) e $36 \%$ eram migrantes ${ }^{25}$. Em 2020, foram ofertadas 200 vagas para o curso de Medicina ${ }^{26}$.

Ao término da consulta, o profissional preenche, além do prontuário, uma planilha em Excel que contém dados sociodemográficos e clínicos de todos os casos atendidos. Quando necessário, como na prescrição de psicofármacos, o psiquiatra acompanha o caso com a psicóloga. O mesmo procedimento ocorre quando o psiquiatra necessita do acompanhamento psicológico de um caso. Um profissional da equipe é responsável pelo monitoramento da entrada dos dados e pela liberação semanal da planilha. Todos os profissionais envolvidos participam de uma reunião semanal na qual os casos são apresentados e discutidos em equipe e supervisionados pelo coordenador do serviço. A duração do projeto será pelo período de tempo estipulado pela reitoria da UFRJ para as aulas de forma remota.

Durante os atendimentos remotos, foi percebido um forte impacto da pandemia na vida dos estudantes. Em um primeiro momento, as demandas caracterizaram-se pelas necessárias adaptações à vida acadêmica e social, como o distanciamento social e o ensino remoto, que potencializaram quadros de ansiedade e depressão. Com o decorrer da pandemia e o adiamento das atividades presenciais, outras demandas emocionais somaram-se a esse contexto: estresse, irritabilidade, frustração, solidão, dificuldades de concentração, sobrecarga de trabalhos e sensação de baixa produtividade acadêmica. Por conta disso, tornou-se essencial fornecer apoio psicossocial a esses estudantes, para que pudessem se reorganizar na nova realidade imposta pela pandemia.

Figura 1. Fluxograma do teleatendimento em saúde mental para estudantes de Medicina da UFRJ durante a pandemia da Covid-19.

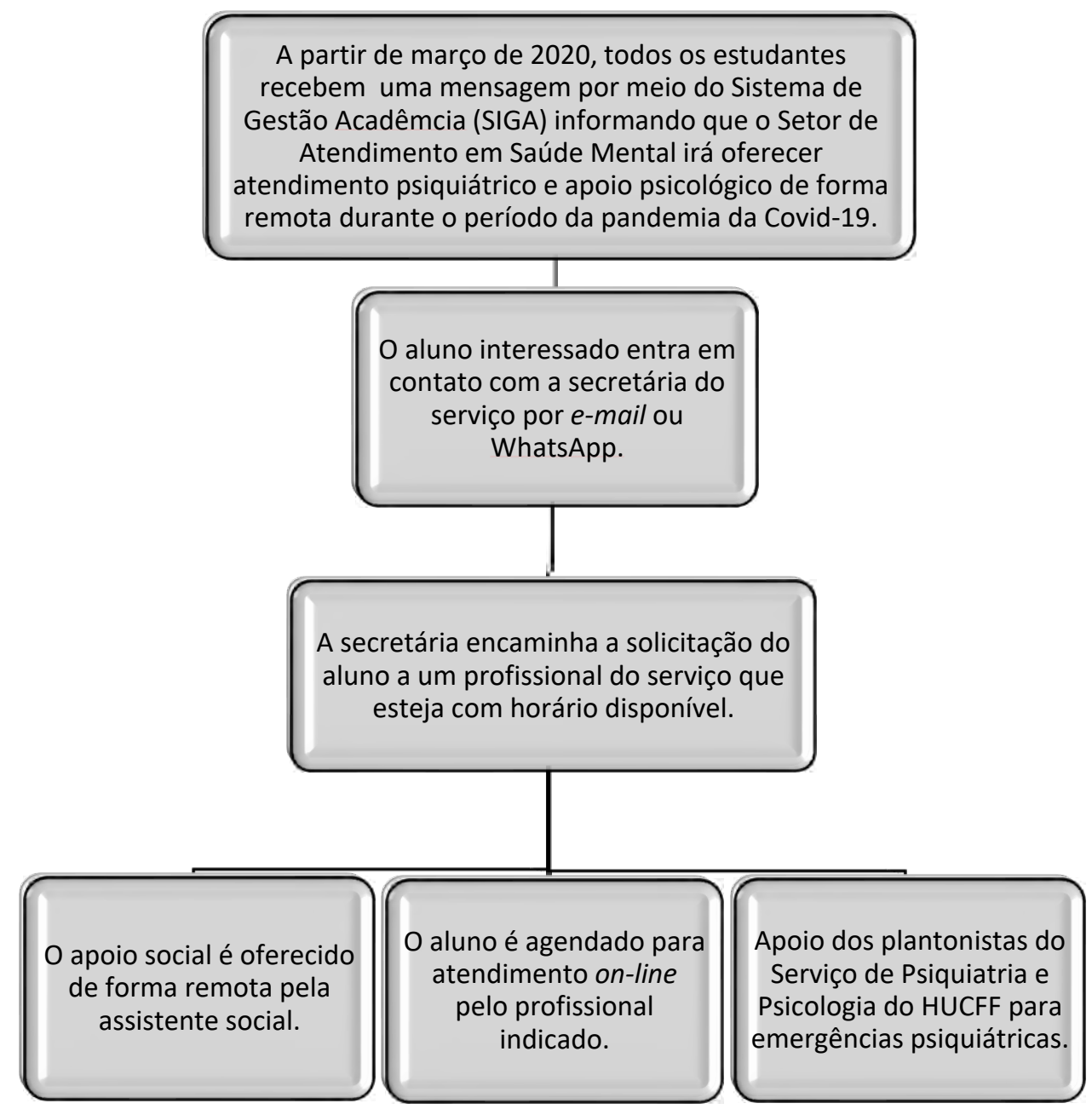

Fonte: Elaborada pelos autores. 


\section{DISCUSSÃO}

A atual reposição das aulas por via remota tornou-se um desafio pelo caráter eminentemente prático de várias disciplinas, sendo fonte de sobrecarga emocional. Esse fato é um dilema principalmente nos dois últimos anos da faculdade (internato) ${ }^{27}$. Além disso, uma parcela significativa desses estudantes apresenta uma maior vulnerabilidade para problemas mentais, como aqueles com renda familiar mais baixa que moram em comunidades carentes e com altos índices de violência ${ }^{28-30}$. Da mesma forma, os migrantes foram, em muitos casos, obrigados a regressar a suas cidades e seus estados na expectativa do retorno às aulas. Lidar com o medo e a insegurança, e conviver com situações de adoecimento, desemprego dos pais e perda econômica são fontes de grande sofrimento psíquico ${ }^{31}$. Nesse contexto, o Setor de Atendimento em Saúde Mental destinado aos alunos de Medicina da UFRJ encontrou no teleatendimento a possibilidade de continuar ofertando cuidado, tendo em vista que esses alunos enfrentam estressores únicos no contexto da pandemia.

O tipo de tecnologia utilizada pode ser um facilitador do atendimento remoto, o que foi observado por meio da utilização de plataformas de fácil manuseio, às quais professores e alunos já estavam habituados. Entretanto, há de se ressaltar que os alunos que habitam em áreas de maior vulnerabilidade tiveram dificuldades de acesso a essas plataformas, o que interferiu na oferta de cuidado ${ }^{32}$. Além da facilidade de adaptação à tecnologia, a existência prévia da estrutura do serviço, com uma equipe de profissionais já formada, fez com que a implementação fosse mais rápida e possibilitou sua manutenção ${ }^{33}$.

Outros facilitadores indicados são a experiência dos profissionais e a familiaridade com os pacientes, o que foi alcançado pelo serviço que contava com a presença de profissionais que já faziam parte da instituição e tinham uma larga experiência no atendimento em saúde mental ${ }^{33}$. Ressaltam-se ainda outros aspectos que contribuem para a implementação adequada do teleatendimento, como economia de custos e diminuição do tempo de espera e do tempo gasto em trânsito para a consulta ${ }^{33}$. Ao experienciar tais pontos, a nossa equipe observou uma economia de custos, tendo em vista que os gastos para implementação foram baixos (graças à existência prévia da estrutura e ao tipo de tecnologia utilizada). Além disso, houve uma redução do tempo de deslocamento e de espera para atendimentos, o que resultou em melhora da acessibilidade e na adequação mais eficiente à extensa carga horária dos estudantes. Um outro importante fator observado foi o apoio institucional, como o oferecido pelo diretor da Faculdade de Medicina, que possibilitou a divulgação do projeto por meio de mensagens pelo SIGA da UFRJ, de modo a alcançar em maior abrangência os alunos.
Chen et al. ${ }^{12}$ apontam a privacidade e a confiança como vantagens do teleatendimento. Entretanto, foi observado que a privacidade pode ter sido comprometida principalmente entre os alunos mais vulneráveis, cujas residências apresentam espaço físico reduzido e nas quais se faz necessário dividir o mesmo ambiente com outros integrantes da família. A falta de privacidade pode interferir, por exemplo, na espontaneidade do relato dos problemas familiares vivenciados por esses alunos.

A relação de confiança pode também ser facilitada pela credibilidade dada pelos alunos aos profissionais da instituição, devido ao seu conhecimento acadêmico na área de saúde mental $^{33}$. Esses profissionais, contudo, encontram-se muitas vezes sobrecarregados e dispõem de pouco tempo para dedicarse aos atendimentos. Apesar de, como mencionado, haver uma maior capacidade de adaptação no formato de atendimento remoto, a extensa carga horária dos estudantes pode dificultar a conciliação de seus horários com os disponibilizados pelos profissionais, o que pode prejudicar a criação de vínculo e a continuação do cuidado.

Entre as barreiras para esse tipo de serviço, estão também as condições de saúde do paciente ${ }^{33}$. Foi identificada uma dificuldade de manejo em situações de emergência psiquiátrica, como em tentativas de suicídio, apesar da existência do serviço de emergência do HUCFF que faz parte do Setor de Atendimento Psiquiátrico e Psicológico, ao qual o estudante poderá ser encaminhado se houver necessidade.

O teleatendimento pode ainda limitar a relação médicopaciente, impedindo uma adequada comunicação não verbal entre eles. É possível que se percam, durante o atendimento virtual, expressões úteis para definição de diagnóstico ou manejo, como percepção de olhares, formas de gesticular as mãos, modo de sentar, entre outras ${ }^{34,35}$.

Além disso, há dois pontos que podem funcionar como barreira para implementação do teleatendimento: a escassez de treinamentos específicos dos profissionais para essa modalidade e a insuficiência de diretrizes que possam auxiliar nesse processo. Com o advento da pandemia, a necessidade de adaptação fez com que a modalidade remota fosse adotada rapidamente, sem que houvesse, em um momento inicial, um treinamento da equipe para a adequação a esse tipo de atendimento ${ }^{36}$.

Assim, pode-se perceber que o teleatendimento, apesar de algumas limitações, tem servido como um espaço importante de escuta e acolhimento diante das demandas psicossociais desses alunos, o que também tem sido evidenciado em pesquisas que demonstram satisfação tanto de profissionais de saúde quanto de paciente com o emprego dessa forma de ofertar cuidado 37,38 . 


\section{CONCLUSÕES}

A implantação do teleatendimento para assistência à saúde no Brasil está ainda em sua fase inicial e precisou ser realizada de forma abrupta por conta das condições e necessidades impostas pela pandemia. O mesmo foi observado na implementação do serviço, mas, apesar das dificuldades inerentes ao processo, percebe-se o potencial da tecnologia em auxiliar a população neste momento crítico, em especial na atenção à saúde mental de grupos específicos como os estudantes de Medicina.

Abre-se, portanto, uma janela de oportunidades de aprendizado e reconfiguração de ofertas e acessos de cuidado, com o potencial de trazer benefícios para o atendimento em saúde mental destinado aos estudantes. O teleatendimento tem o desafio de ultrapassar algumas barreiras que dificultam o acesso e a disponibilidade de serviço de saúde mental no campus universitário. Entretanto, a integração entre o atendimento presencial e o teleatendimento pode aperfeiçoar o cuidado em saúde mental no período posterior à pandemia. O desenvolvimento de pesquisas torna-se também importante na avaliação da aceitabilidade e viabilidade dessas novas tecnologias, com a perspectiva da expansão desse cuidado para outros cursos da UFRJ.

\section{CONTRIBUIÇÃO DOS AUTORES}

Suzana Pacheco Liberal e Geovani Bordiano participaram da concepção, redação e editoração final do artigo. Giovanni Marcos Lovisi e Lucia Abelha participaram da concepção, redação e revisão do artigo. Fernanda Monteiro Dias, Camila Oliveira Carvalho, Lina Rosa Nunes Morais e Marco Antonio Alves Brasil participaram da redação e revisão do artigo.

\section{CONFLITO DE INTERESSES}

Declaramos não haver conflito de interesses.

\section{FINANCIAMENTO}

Declaramos não haver financiamento.

\section{REFERENCES}

1. Thakur V, Jain A. Covid 2019-suicides: a global psychological pandemic. Brain Behav Immun. 2020;88:952-3. doi: 10.1016/j.bbi.2020.04.062.

2. Gunnell D, Appleby L, Arensman E, Hawton K, John A, Kapur N, et al. Suicide risk and prevention during the Covid-19 pandemic. Lancet Psychiatry. 2020;7:468-71. doi: 10.1016/S2215-0366(20)30171-1.

3. Brasil. Recomendações gerais. Saúde mental e atenção psicossocial na pandemia Covid-19. Brasília: Ministério da Saúde, Fiocruz; 2020 [access in 25 jun 2021]. Available from: https://www.fiocruzbrasilia. fiocruz.br/wp-content/uploads/2020/04/Sa\%c3\%bade-Mentale-Aten \%c3\%a7\%c3\%a3o-Psicossocial-na-Pandemia-Covid-19recomenda\%c3\%a7\%c3\%b5es-gerais.pdf.
4. Shigemura J, Ursano RJ, Morganstein JC, Kurosawa MBD. Public responses to the novel 2019 coronavirus (2019-nCoV) in Japan: mental health consequences and target populations. Psychiatry Clin Neurosci. 2020;74:281-2. doi: $10.1111 /$ pcn. 1298.

5. Baker DM, Bhatia S, Brown S, Cambridge W, Kamarajah SK, McLean KA et al. Medical student involvement in the Covid-19 response. Lancet. 2020;395:1254. doi: 10.1016/S0140-6736(20)30795-9.

6. Rotenstein LS, Ramos MA, Torre M, Segal BJ, Peluso MJ, Guille C, et al Prevalence of depression, depressive symptoms, and suicidal ideation among medical students a systematic review and meta-analysis. J Am Med Assoc. 2016;316(21):2214-36. doi: 10.1001/jama.2016.17324.

7. Ornell F, Schuch JB, Sordi AO, Kessler FHP. "Pandemic fear" and Covid-19: mental health burden and strategies. Brazilian J Psychiatry. 2020;1-5. doi: 10.1590/1516-4446-2020-0008.

8. Catapan SC, Calvo MCM. Teleconsulta: uma revisão integrativa da interação médico-paciente mediada pela tecnologia. Rev Bras Educ Med. 2020;44(1):1-13. doi: 10.1590/1981-5271v44.1-20190224.

9. Brasil. Suicídio na pandemia Covid-19. Saúde mental e atenção psicossocial na pandemia Covid-19. Brasília: Ministério da Saúde, Fiocruz; 2020 [access in 25 jun 2021]. Available from: https://www.fiocruzbrasilia.fiocruz.br/ wpcontent/uploads/2020/05/cartilha_prevencaosuicidio.pdf15.

10. Caetano R, Silva AB, Guedes ACCM, Paiva CCN, Ribeiro GR, Santos DL, et al. Desafios e oportunidades para telessaúde em tempos da pandemia pela Covid-19: uma reflexão sobre os espaços e iniciativas no contexto brasileiro. Cad Saude Publica. 2020;36(5):e00088920. doi: 10.1590/0102$311 \times 00088920$.

11. Declaração de Tel Aviv sobre responsabilidades e normas éticas na utilização da telemedicina. 2003 [access in 25 jun 2021]. Available from: https://portal.cfm.org.br/artigos/responsabilidades-e-normas-eticas-nautilizacao-da-telemedicina/.

12. Chen JA, Chung W, Young SK, Tuttle MC, Collins MB, Darghouth SL, et al. Covid-19 and telepsychiatry: early outpatient experiences and implications for the future. Gen Hosp Psychiatry. 2020 July;66:89-95. doi: 10.1016/j.genhosppsych.2020.07.002.

13. Zhou $X$, Snoswell CL, Harding LE, Bambling M, Edirippulige $S$, Bai $X$, et al. The role of telehealth in reducing the mental health burden from Covid-19. Telemed e-Health. 2020;26(4):377-9. doi: 10.1089/tmj.2020.0068.

14. Dias RS, Marques AFH, Diniz PRB, Silva TAB, Cofiel L, Mariani MMC, et al. Telemental health in Brazil: past, present and integration into primary care. Rev Psiquiatr Clín. 2015;42(2):41-4. doi: 10.1590/0101-60830000000046.

15. TelePSI Covid-19: cuidando da linha de frente. 2021 [access in 25 jun 2021]. Available from: https://sites.google.com/hcpa.edu.br/telepsi/ in\%C3\%ADcio?authuser $=0$.

16. Brasil. Portaria n 467, de 20 de março de 2020. Dispõe, em caráter excepcional e temporário, sobre as ações de Telemedicina, com o objetivo de regulamentar e operacionalizar as medidas de enfrentamento da emergência de saúde pública de importância internacional previstas no art. $3^{\circ}$ da Lei $n^{\circ} 13.979$, de 6 de fevereiro de 2020, decorrente da epidemia de COVID-19. Diário Oficial da União; 2020 [access in 25 jun 2021]. Available from: https://www.in.gov.br/en/web/dou/-/portaria-n-467-de20-de-marco-de-2020-249312996.

17. Brasil. Lei no 13.989 , de 15 de abril de 2020. Dispõe sobre o uso da telemedicina durante a crise causada pelo coronavírus (SARS-CoV-2) Diário Oficial da União; 2020 [access in 25 jun 2021]. Available from: https://www.in.gov.br/en/web/dou/-/lei-n-13.989-de-15-de-abrilde-2020-252726328.

18. Conselho Federal de Medicina. Ofício CFM № 1756/2020. CFM, 2020 [access in 25 jun 2021]. Available from: https://portal.cfm.org.br/images/ PDF/2020_oficio_telemedicina.pdf.

19. Conselho Federal de Psicologia. Nova resolução do CFP orienta categoria sobre atendimento on-line durante pandemia da Covid-19. CFP; 2020 [access in 25 jun 2021]. Available from: https://site.cfp.org.br/novaresolucao-do-cfp-orienta-categoria-sobre-atendimento-on-line-durantepandemia-da-covid-19/. 
20. Conselho Federal de Serviço Social. CFESS divulga nota sobre o exercício profissional diante da pandemia do Coronavírus. Cfess; 2020 [access in 25 jun 2021]. Available from: http://www.cfess.org.br/visualizar/noticia/ $\operatorname{cod} / 1679$.

21. Universidade Federal do Rio de Janeiro. Boletim $n^{\circ} 46$, de 16 de novembro de 2017 [aceso em 25 jun 2021]. Available from: http://siarq.ufrj.br/ images/bufrj/2017/46-2017.pdf.

22. Universidade Federal do Rio de Janeiro. Boletim $n^{\circ} 10$, de 7 de março de 2019 [access in 25 jun 2021]. Available from: http://siarq.ufrj.br/images/ bufrj/2019/10-2019.pdf.

23. Pacheco JPG, Giacomin HT, Tam WW, Ribeiro TB, Arab C, Bezerra IM, et al. Mental health problems among medical students in Brazil: a systematic review and meta-analysis. Rev Bras Psiquiatr. 2017;39(4):369-78. doi: 10.1590/1516-4446-2017-2223.

24. Morganstein JC, Ursano RJ. Ecological disasters and mental health: causes, consequences, and interventions. Front Psychiatry. 2020;11:1-15. doi: 10.3389/fpsyt.2020.00001.

25. Universidade Federal do Rio de Janeiro. Plano de Desenvolvimento Institucional 2012 a 2023: informações institucionais - reitoria. Rio de Janeiro: UFRJ; 2018 [access in 25 jun 2021]. Available from: https:// docplayer.com.br/86793806-Plano-de-desenvolvimento-institucional2012-a-2023-informacoes-institucionais-reitoria.html.

26. Universidade Federal do Rio de Janeiro. Termo de Adesão UFRJ - SiSU MEC 2020/1. 2019 [access in 25 jun 2021]. Available from: https:// acessograduacao.ufrj.br/2020-1/2020-1-sisu-mec/termo-de-adesao-ufrjsisu-mec-2020-1.

27. Salum GA, Rehmenklau JF, Csordas MC, Pereira FP, Castan JU, Ferreira AB, et al. Supporting people with severe mental health conditions during the Covid-19 pandemic: considerations for low- and middle-income countries using telehealth case management. Braz J Psychiatry. 2020;42(4):451-2. doi: 10.1590/1516-4446-2020-1078.

28. Gonçalves DA, Mari JJ, Bower P, Gask L, Dowrick C, Tófoli LF, et al. Estudo multicêntrico brasileiro sobre transtornos mentais comuns na atenção primária: prevalência e fatores sociodemográficos relacionados. Cad Saude Publica. 2014;30:623-32. doi: 10.1590/0102-311X00158412.

29. Barros FC, Matijasevich A, Santos IS, Horta BL, Silva BGC, Munhoz TN, et al. Social inequalities in mental disorders and substance misuse in young adults: a birth cohort study in Southern Brazil. Soc Psychiatry Psychiatr Epidemiol. 2018;53:717-26. doi: 10.1007/s00127-018-1526-x.
30. Patel V, Kleinman A. Poverty and common mental disorders in developing countries. Bull World Health Organ. 2003;81:609-15. doi: 10.1590/S004296862003000800011

31. Alsoufi A, Alsuyihili A, Msherghi A, Elhadi A, Atiyah H, Ashini A, et al. Impact of the Covid-19 pandemic on medical education: medical students' knowledge, attitudes, and practices regarding electronic learning. PLoS One. 2020 Nov;15(11):e0242905. doi: 10.1371/journal.pone.0242905.

32. Muir SD, Boer K, Nedeljkovic M, Meyer D. Barriers and facilitators of videoconferencing psychotherapy implementation in veteran mental health care environments: a systematic review. BMC Health Serv Res. 2020;20:999. doi: 10.1186/s12913-020-05858-3.

33. Almathami HKY, Win KT, Vlahu-Gjorgievska E. Barriers and facilitators that influence telemedicine-based, real-time, online consultation at patients' homes: systematic literature review.J Med Internet Res. 2020;22(2):e16407. doi: 10.2196/16407.

34. Cosenza TRSB, Pereira ER, Silva RMCRA, Medeiros AYBBV. Desafios da telepsicologia no contexto do atendimento psicoterapêutico online durante a pandemia de Covid-19. Research, Society and Development. 2021;10(4):e52210414482. doi: 10.33448/rsd-v10i4.14482.

35. Luz PL. Telemedicine and the doctor/patient relationship. Arq Bras Cardiol. 2019;113(1):100-2. doi: 10.5935/abc.20190117.

36. Diwan MN, Awan HA, Aamir A, Filippis R, Ullah I. Telepsychiatry in lowand middle-income countries during Covid-19: pandemic, barriers, and road model. J Nerv Ment Dis. 2021;209(2):144-6. doi: 10.1097/ NMD.0000000000001245.

37. Andrews G, Cuijpers P, Craske MG, McEvoy P, Titov N. Computer therapy for the anxiety and depressive disorders is effective, acceptable and practical health care: a meta-analysis. PLoS One. 2010;5(10):e13196. doi: 10.1371/ journal.pone.0013196.

38. Connolly SL, Miller CJ, Lindsay JA, Bauer MS. A systematic review of providers' attitudes toward telemental health via videoconferencing. Clin Psychol Sci Pract. 2020;00:e12311. doi: 10.1111/cpsp.1231. 